\title{
Polypharmacy in Clinical Practice
}

\author{
Shalini Gupta \\ University of Dundee Nethergate Dundee Scotland, UK
}

*Corresponding Author: Shalini Gupta, University of Dundee Nethergate Dundee Scotland, UK

\section{INTRODUCTION}

Pharmacotherapy is undoubtedly the most common clinical intervention for many disease conditions. While it can be highly effective in treating diseases or slowing disease progression, there are often concerns raised regarding the inappropriate use of drugs. This can lead to adverse drug reactions (ADR) and drug-drug interactions (DDI). The term polypharmacy means "multiple medications" and is considered to be present when a patient takes five or more medications. It may be "appropriate polypharmacy" when all drugs are prescribed for specific therapeutic objectives and the drug therapy has been optimised to minimise the risk of ADR. However, on occasions "inappropriate polypharmacy" might be present, when one or more drugs are prescribed that are no longer needed, and the combination of several drugs puts the patient at an unacceptably high risk of ADR.

Inappropriate polypharmacy is a clinical concern since the risks due to medications outweigh benefits for the individual patient. Rational prescribing should address this through identifying patients at greater risk of harm and deciding a medication regimen that is tailored to their changing needs and expectations. The importance of polypharmacy management is recognised worldwide. The recent WHO Third Global Patient Safety Challenge "Medication without Harm" aims to reduce severe avoidable medication related harm by $50 \%$ over 5 years, globally [1]. The EU funded project SIMPATHY [2] has been studying polypharmacy management in Europe and has identified recommendations to improve medication safety.

\section{Prescribing Cascade}

Polypharmacy can be frequently a consequence of "prescribing cascade". Prescribing cascades result when ADR to medications are treated symptomatically as newly developed conditions owing to not being recognised as drug-induced. This symptomatic prescribing leads to increase in the number of drugs per prescription and "pill burden". Eventually, the cumulative effect of multiple drugs has the potential to lead to serious complications. Table 1 gives examples of some common prescribing cascades in clinical practice.

Table1. Common clinical prescribing cascades

\begin{tabular}{|c|c|c|}
\hline Initial drug & Resulting ADR & Symptomatic treatment \\
\hline Amitriptyline & Decreased cognition & Galantamine \\
\hline Proton pump inhibitor & Vitamin B12 deficiency & Vitamin supplementation \\
\hline Donepezil & Urinary incontinence & Oxybutynine \\
\hline Amlodipine & Ankle edema & Furosemide \\
\hline Prochloroperazine & Parkinsonism & Levodopa \\
\hline Prednisolone & Diabetes & Oxybutynin \\
\hline Furosemide & Urinary frequency & \\
\hline
\end{tabular}

It is important to recognise that new conditions that develop in patients might be drug induced and explore if there is potential to stop the offending medication. Other pharmacological or nonpharmacological alternatives may be considered in place of the suspected drug. Targeted deprescribing (discussed below) in chronic conditions avoids ADRs and improves clinical outcomes. Thus, recognising and avoiding prescribing cascades in practice can reduce medication burden and improve quality of life. 


\section{Polypharmacy in the Elderly}

Polypharmacy is a common problem encountered in all clinical settings but it is of particular concern in the elderly patients due to additional risk factors. Apart from causing ADR and DDI, polypharmacy can lead to increased risk of non-adherence, cognitive impairment, risk of falls, increased morbidity, hospitalizations and mortality in the elderly $[3,4]$. The reasons for these polypharmacy related complications in the elderly are manifold. Pharmacokinetics and pharmacodynamics change as people age due to progressive functional decline in organ systems.

There are changes in a number of pharmacokinetic parameters with aging. There is an age-related decrease in small-bowel surface area, slowed gastric emptying, and an increase in gastric $\mathrm{pH}$ leading to reduced absorption of drugs that require an acidic $\mathrm{pH}$ (eg calcium carbonate). There is a relative increase in body fat and reduction in lean body mass. The increase in fat increases the volume of distribution of highly lipophilic drugs (eg, diazepam, chlordiazepoxide). This leads to an increase in their elimination half-lives causing a risk of toxicities. Reduced albumin levels are common in elderly which can result in toxicity of highly protein bound drugs such as warfarin and phenytoin [5].

Hepatic metabolism of many drugs through the cytochrome P-450 enzyme system decreases with age leading to a reduction in clearance by upto $30-40 \%$. Thus, drug dosage should be decreased accordingly and dose adjustments should be individualized. The most important pharmacokinetic change associated with aging is decreased renal elimination of drugs. The age-related decrease varies substantially from person to person and creatinine clearance (measured or estimated using computer programs or a formula, such as Cockcroft-Gault) is used to guide dosing for most drugs eliminated by the kidneys [6].

Pathological age-associated changes in organs can lead to changes in drug-receptor interaction, in post-receptor events, and in adaptive homeostatic responses among frail patients. Older people's central nervous system is often more sensitive to opioids, benzodiazepines, anti-parkinson medications and anti-psychotics. The elderly are also particularly sensitive to anticholinergic drug effects. Many drugs (eg, tricyclic antidepressants, sedating antihistamines, urinary antimuscarinic agents, some antipsychotic drugs, antiparkinsonian drugs with atropine-like activity, many OTC hypnotics and cold preparations) have anticholinergic properties. Elderly patients, particularly those with cognitive impairment, are particularly prone to CNS adverse effects of such drugs and may become more confused and drowsy [7]. Antimuscarinic effect of drugs can also cause constipation, urinary retention (especially in older men with benign prostatic hyperplasia), blurred vision, orthostatic hypotension, and dry mouth. It is recommended to reduce anticholinergic burden on patients, this category of drugs should be avoided if possible and if used, doses should be minimised [8]. There is insufficient evidence related to appropriate medication use in the geriatric age group. It should be borne in mind that even though the elderly population are the biggest consumer of drugs, they are not represented in the clinical trials. Various strategies have been developed to reduce risk of ADR in the elderly- eg Screening Tool of Older Person's Prescriptions (STOPP) and Screening Tool to Alert doctors to Right Treatment (START) [9]. STOPP consists of 65 clinically significant criteria for inappropriate prescribing in the elderly and START comprises of 22 evidence-based prescribing indicators for frequently encountered disease conditions in the geriatric population. The Beers List of potentially inappropriate medications for older adults is another frequently used reference tools in the field of geriatrics.

\section{PolypharmaCY AND ECONOMiC BURdeN}

Polypharmacy increases the economic burden on healthcare resources directly through greater number of drugs dispensed and consumed. There is increase in the cost of prescribing and medicines waste. A patient receiving multiple medicines requires more monitoring and greater contact with health professionals. There is also the additional expense of unscheduled hospital admissions due to ADR and polypharmacy complications. SIMPATHY Economic Analysis tool [10] has been developed as a part of European commission project to analyse the costs and benefits associated with carrying out polypharmacy reviews. It has the potential to be included in the polypharmacy management plan to improve resource productivity.

\section{REVIEWING MEDICATION NEED AND EFFECTIVENESS}

Numbers needed to treat (NNT) are calculated to understand the probable clinical efficacy of a drug for the individual patient and to predict the likely impact over a 12 month period. NNT is an objective 
measure of the effectiveness of a particular medication or intervention. The NNT is the average number of patients who require to be treated for one to benefit compared with a control in a clinical trial. Annualised NNT provides a numerical comparison between different therapeutic options. The ideal NNT is 1 , when every treated person improves with the treatment. The higher the NNT, the less effective is the treatment.

Clinicians may consider discussing the NNT for a range of medicines available for a particular condition as a strategy to aid in therapeutic decision making with the multidisciplinary team as well as with patients about the likely benefit [11].

\section{DEPRESCRIBING}

Deprescribing is the planned and supervised process of dose reduction or stopping of medication that might be causing harm, or no longer be of benefit. It is part of good prescribing and implies reducing doses when they are too high or stopping medications that are no longer needed.

Clinicians and pharmacists can undertake a medication review for patients taking multiple drugs and attempt deprescribing which has been found to be a safe and effective approach towards addressing polypharmacy. Optimizing medication through targeted deprescribing is a vital part of managing chronic conditions, avoiding adverse effects and improving outcomes. The goal of deprescribing is to reduce medication burden and maintain or improve quality of life.

Decisions around deprescribing can be very difficult. Physicians report various challenges in weighing the benefits and harms of continuing or stopping medications, as well as identify a need to have clear information about the processes for cessation in order to gain confidence regarding deprescribing [12]. Few evidence-based guidelines exist to support safe deprescribing for specific classes of medication. These include proton pump inhibitors, benzodiazepines, antipsychotics, dementia medications among others. The guidelines incorporate easy to use algorithms that provide evidence for the benefits and harms of continuing or deprescribing the medications. These algorithms also take into account patient preferences and values surrounding deprescribing, together with practical advice on how to implement deprescribing [13]. Medstopper is another useful strategy developed to aid deprescribing. It is a web-based tool developed by a team of health professionals to help doctors and their patients look at a list of medications to decide if some should be stopped or changed. The medications are ranked in order with those least safe or effective at the top (consider stopping first) and colour coded. Educational resources are available that include case reports and examples of polypharmacy management and deprescribing [14,15].

\section{CONCLuSion}

Polypharmacy is a growing problem with patients with multi-morbidities and the geriatric population particularly at risk. This paper highlights the risks and consequences of prescribing cascades in clinical practice and strategies to avoid them. Various screening tools to detect inappropriate polypharmacy and educational resources relevant to clinicians, pharmacists and nursing prescribers are included. Pragmatic measures that the healthcare professionals might consider in their regular day to day practice have been discussed with a particular focus on deprescribing high-risk medications in the elderly.

\section{REFERENCES}

[1] World Health Organization. Quality of care: a process for making strategic choices in health systems. Geneva: World Health Organization; 2006. 4

[2] Mair A, Fernandez-Limos F, Alonso A, Harrison C, Hurding S, et al. Polypharmacy Management by 2030: a patient safety challenge, 2nd Edition. Coimbra: SIMPATHY Consortium, 2017. http://www.simpathy.eu/ sites/default/files/Managing_polypharmacy2030web.pdf

[3] Kwan D. Polypharmacy: optimizing medication use in elderly patients. Practice. 2013 Apr;20:25.

[4] Shah BM, Hajjar ER. Polypharmacy, adverse drug reactions, and geriatric syndromes. Clinics in geriatric medicine. 2012 May 1;28(2):173-86.

[5] ELDesoky ES. Pharmacokinetic-pharmacodynamic crisis in the elderly. American journal of therapeutics. 2007 Sep 1;14(5):488-98.

[6] Aymanns C, Keller F, Maus S, Hartmann B, Czock D. Review on pharmacokinetics and pharmacodynamics and the aging kidney. Clinical Journal of the American Society of Nephrology. 2010 Jan 7:CJN-03960609. 
[7] Bishara D, Harwood D, Sauer J, Taylor D. Anticholinergic effect on cognition (AEC) of drugs commonly used in older people. International Journal of Geriatric Psychiatry. 2016;32(6):650-656

[8] Sumukadas D, McMurdo M, Mangoni A, Guthrie B. Temporal trends in anticholinergic medication prescription in older people: repeated cross-sectional analysis of population prescribing data. Age and Ageing. 2013;43(4):515-521

[9] Hill-Taylor B, Sketris I, Hayden J, Byrne S, O'sullivan D, Christie R. Application of the STOPP/START criteria: a systematic review of the prevalence of potentially inappropriate prescribing in older adults, and evidence of clinical, humanistic and economic impact. Journal of clinical pharmacy and therapeutics. 2013 Oct;38(5):360-72.

[10] Stewart D, Mair A, Wilson M, Kardas P, Lewek P, Alonso A, McIntosh J, MacLure K, SIMPATHY consortium. Guidance to manage inappropriate polypharmacy in older people: systematic review and future developments. Expert opinion on drug safety. 2017 Feb 1;16(2):203-13.

[11] Leucht S, Hierl S, Kissling W, Dold M, Davis JM. Putting the efficacy of psychiatric and general medicine medication into perspective: review of meta-analyses. The British Journal of Psychiatry. 2012 Feb;200(2):97-106.

[12] Anderson K, Stowasser D, Freeman C, Scott I. Prescriber barriers and enablers to minimising potentially inappropriate medications in adults: a systematic review and thematic synthesis. BMJ Open. 2014;4(12)

[13] Tannenbaum C, Martin P, Tamblyn R, Benedetti A, Ahmed S. Reduction of Inappropriate Benzodiazepine Prescriptions Among Older Adults Through Direct Patient Education: The EMPOWER Cluster Randomized Trial. JAMA Intern Med. 2014; 174(6):890-898.

[14] Farrell B, Shamji S, Monahan A, French Merkley V. Clinical vignettes to help you deprescribe medications in elderly patients: introduction to the polypharmacy case series. Canadian Family Physician 2013;59:1257-1258.

[15] Farrell B, Shamji S, Monahan A, French Merkley V. Reducing polypharmacy in the elderly: Cases to help you rock the boat. Canadian Pharmacists Journal 2013;146(5):243-244.

Citation: Shalini Gupta, Dr. (2019). Polypharmacy in Clinical Practice. ARC Journal of Pharmaceutical Sciences (AJPS), 5(1), pp.1-4. http://dx.doi.org/10.20431/2455-1538.0501001

Copyright: (C) 2019 Authors. This is an open-access article distributed under the terms of the Creative Commons Attribution License, which permits unrestricted use, distribution, and reproduction in any medium, provided the original author and source are credited. 